\title{
The Effective Flexural Modulus of Filament Wound GRP Tapered Poles
}

\author{
Ana SKENDER*, Zoran DOMITRAN, Jakov KROKAR
}

\begin{abstract}
The composite lighting poles are filament wound GRP tapered tubes with a variable wall thickness across the length. In this study, the effective flexural properties of GRP poles have been determined, experimentally and theoretically. Cantilever bending tests were performed on full-scale GRP tubes and the effective flexural modulus was calculated from the initial slope of the flexural stress-strain curve based on the classical beam theory. The results show that the strain range corresponding to the linear behaviour is the same as under three-point and four-point tests with smaller specimens. Unlike other methods, the proposed method considers the variation of the crosssectional properties across the pole length. The effective properties of GRP tubes were further determined considering the orthotropic and unsymmetrical layup of the wall segment. A sensitivity analysis was performed at the lamina as well as the laminate level in order to evaluate the influence of material properties of the matrix and the fibres, the amount and orientation of the fibres as well as the layer thickness. Finally, the effective (replacement) flexural modulus of a filament wound tube was determined based on the classical lamination theory and compared to experimentally determined values. It was concluded that the replacement flexural modulus is a matrix dominated property highly influenced by the fibre volume fraction.
\end{abstract}

Keywords: cantilever bending test; classical beam theory; classical lamination theory; effective flexural modulus; fibre reinforced polymer pole; filament winding; tapered tube

\section{INTRODUCTION}

Composite lighting poles made of fibre reinforced polymer (FRP) have recently become an excellent alternative to steel and concrete lighting poles. Lighting poles are used to support brackets and luminaires in public lighting systems. The most important advantages of using FRP lighting poles are small weight, resistance to corrosion, electrical safety and good mechanical properties. Nevertheless, a long-term exposure of unprotected FRP materials to ultraviolet radiation can have a negative impact on the aesthetic qualities and mechanical properties. Polymer based coating systems are used to prevent the degradation of mechanical properties [1].

FRP poles are manufactured by the filament winding process and are tapered tubes. Performance classes of FRP poles are related to the requirements of resistance to lateral (wind) loads and performance under vehicle impact [2, 3]. Therefore, experimental testing on poles is usually performed in order to investigate flexural properties [4]. Three-point and four-point flexural tests with small rectangular test specimens are widely accepted for determining the flexural properties of the FRP material [5, $6]$. The flexural modulus is calculated from the initial slope of the $\sigma_{f}-\varepsilon_{f}$ curve based on the classical beam theory considering the simple supported composite beam loaded with one or two concentrated forces [7,8].

FRP poles are laminated composites made of plies (laminas) bonded together. This material is considered a quasi-homogenous material and it is convenient to determine the effective material properties or stiffnesses in the global coordinate system. The effective properties, i.e. engineering constants can be determined at the lamina, laminate and structure level [9].

Polyzois et al. treated the thin wall of a tapered FRP pole as a laminated orthotropic plate in order to determine the effective longitudinal modulus using the classical lamination theory (CLT) [10]. The same value was used for the axial as well as the flexural problem. Chan and Demirhan developed new approaches based on laminated plate and shell theories for calculating the bending stiffness of a composite tube [11]. Both approaches considered the change of the fibre orientation along the circumference. Kollár and Pluzsik specifically addressed thin-walled open and closed section composite beams with orthotropic and unsymmetrical layup [12].

In order to determine the global effective longitudinal modulus, simple approximation methods were developed by summing the effective properties of each group of layers constructing the wall of a composite tube [13, 14]. Metiche and Masmoudi used a similar method in order to determine the bending stiffness and propose the design procedures for the flexural behaviour of glass reinforced polymer (GRP) composite poles [15].

The effective properties of FRP materials are mostly used in order to develop material models in the numerical analysis of FRP tubes [16-18]. They are also used together with the classical beam theory in order to simplify the complex mechanical behaviour of laminated composites. Under small deformations, the response of orthotropic composite beams under different loads can be obtained by replacing the isotropic stiffnesses with the replacement stiffnesses in the corresponding isotropic beam solution [19]. The replacement stiffnesses and consequently the effective moduli are determined at the laminate or structure level by using the CLT. Several researchers have used the effective properties approach for the static and dynamic analysis of GRP poles under different loads [20-23]. Metiche and Masmoudi derived the analytical deflection line of tapered FRP poles based on the classical beam theory by dividing the length of the pole into three zones with different stacking sequences and average thicknesses [24]. The effective longitudinal modulus of each zone was determined by summing the effective properties of each layer and a good agreement was achieved of theoretical values and experimental results from the full-scale cantilever testing on tapered GRP poles.

One of the objectives of this research is to determine the effective flexural modulus of GRP poles, experimentally and theoretically. For that reason, an experimental bending test was carried out on full-scale composite cantilever beams and the deflection was measured. The effective flexural modulus is determined at the characteristic cross-sections based on the comparison 
between experimental results and analytical expressions according to the classical beam theory. Defining analytical expressions for tapered tubes with a variable wall thickness across the length represented a challenge. The effective properties of the FRP material were also derived based on the CLT. A sensitivity analysis was performed at the lamina as well as the laminate level in order to evaluate the influence of material properties of the matrix and the fibres, the amount and orientation of the fibres as well as the layer thickness on the effective properties. Finally, the replacement flexural modulus was determined at the characteristic cross-sections considering the orthotropic and unsymmetrical layup of the wall segment.

The paper is structured as follows. The outline of the methodology is shown in Section 2 with the description of the experimental testing and the determination of the flexural stiffness according to the classical beam theory as well as the CLT. The results are summarized in Section 3. Section 3.1 deals with the determination of the effective flexural modulus based on the classical beam theory and experimental results. Section 3.2 offers the evaluation of the influence of different parameters on the effective properties at the lamina and laminate level. The replacement flexural moduli of FRP poles based on the CLT were compared to the experimental values and evaluated in Section 3.3. Section 4 provides a discussion and Section 5 presents the conclusions.

\section{METHODS}

In this study, the GRP lighting poles were manufactured by the filament winding process. This technology involves winding fibres under tension over a rotating mandrel and is suitable for the manufacturing of composite tubes. The filaments used are E-Glass fibres with the filament diameter of $16 \mu \mathrm{m}$ (Johns Manville StarRov ${ }^{\circledR} 086$ 2400) impregnated in an unsaturated polyester (UP) resin bath (DISTITRON ${ }^{\circledR} 5119$ ESX20Q). The average volume fraction of glass fibres in the composite is $60 \%$, according to the manufacturer. The helical winding method was applied with the winding angle of $\pm 50^{\circ}$ according to the longitudinal axis of the tube. A 1,2 $\mathrm{mm}$ thick finishing layer was also added with the winding angle of $\pm 80^{\circ}$. Before installation, lighting poles are painted with a gelcoat $\left(\mathrm{NORPOL}^{\circledR} \mathrm{GO} 180064 \mathrm{H} \mathrm{W}\right)$ to prevent the degradation of mechanical properties of the polyester resin during usage.

\subsection{Experimental Setup, Procedure and Data}

In order to investigate flexural properties of composite poles, experimental cantilever bending test was carried out on five full-scale poles with length $(L)$ ranging from 4 to 6 $\mathrm{m}$. The GRP poles were subjected to a lateral displacement at the free end by means of a universal static testing machine (Fig. 1). A strain rate of $20 \mathrm{~mm} / \mathrm{min}$ was used under displacement control. During loading, the applied force was measured as well as the deflection at three different measuring points $x=a, x=2 a$ and $x=L$ (Tab. 1). The deflection at measuring points within the span was measured with inductive displacement transducers (Fig. 2).

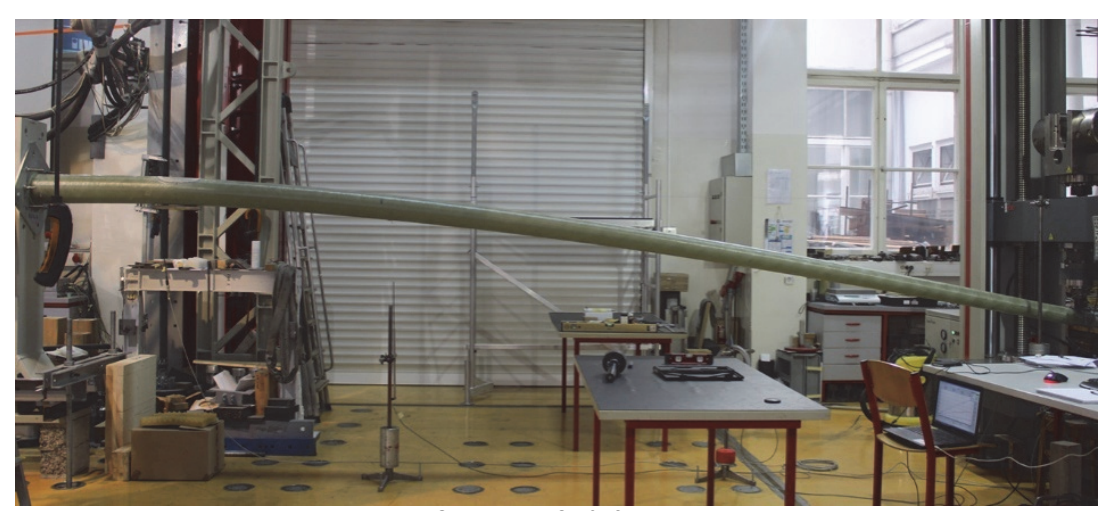

Figure 1 GRP pole RSD6_2 under bending

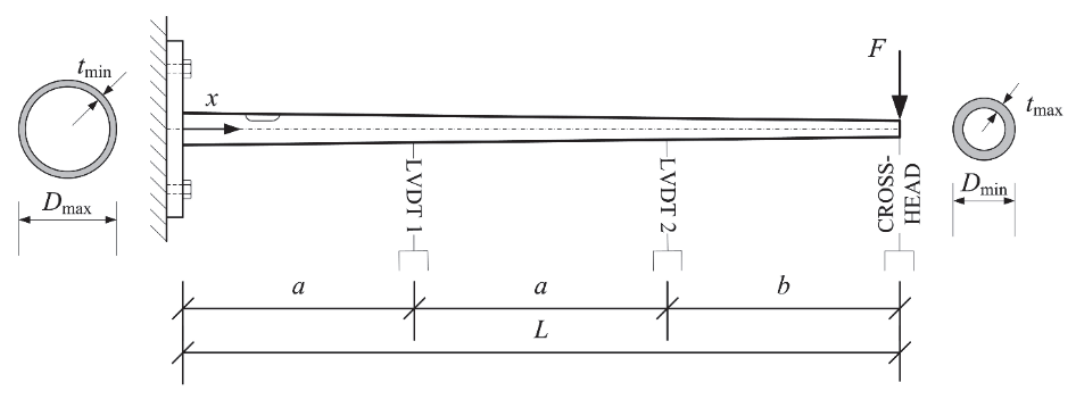

Figure 2 The disposition of measuring points

The tested poles are tapered tubes with different wall thicknesses and each with a variable wall thickness across the length. The maximum outer diameter $D_{\max }$ and minimum wall thickness $t_{\min }$ are located at the fixed end of the pole (Tab. 1). The minimum outer diameter $D_{\min }$ and maximum wall thickness $t_{\max }$ are located at the free end of the pole. 
Table 1 Dimensions of composite poles

\begin{tabular}{|c|c|c|c|c|c|}
\hline $\begin{array}{c}\text { Pole } \\
\text { designation }\end{array}$ & RSD4_1 & RSD5_1 & RSD6_1 & RSD6_2 & RSD6_3 \\
\hline$D_{\max } / \mathrm{mm}$ & 150 & 160 & 165 & 165 & 170 \\
\hline$t_{\min } / \mathrm{mm}$ & 5,5 & 10 & 8 & 7,5 & 10 \\
\hline$D_{\min } / \mathrm{mm}$ & 76 & 100 & 98 & 95 & 104 \\
\hline$t_{\max } / \mathrm{mm}$ & 15,5 & 18 & 18 & 17 & 22 \\
\hline$L / \mathrm{mm}$ & 4000 & 5000 & 6000 & 6000 & 6000 \\
\hline$a / \mathrm{mm}$ & 1500 & 2000 & 2000 & 2000 & 2000 \\
\hline$b / \mathrm{mm}$ & 1000 & 1000 & 2000 & 2000 & 2000 \\
\hline
\end{tabular}

The measured values of the deflection $(w)$ of the free end and the applied force $(F)$ are shown in Fig. 3 for all poles.

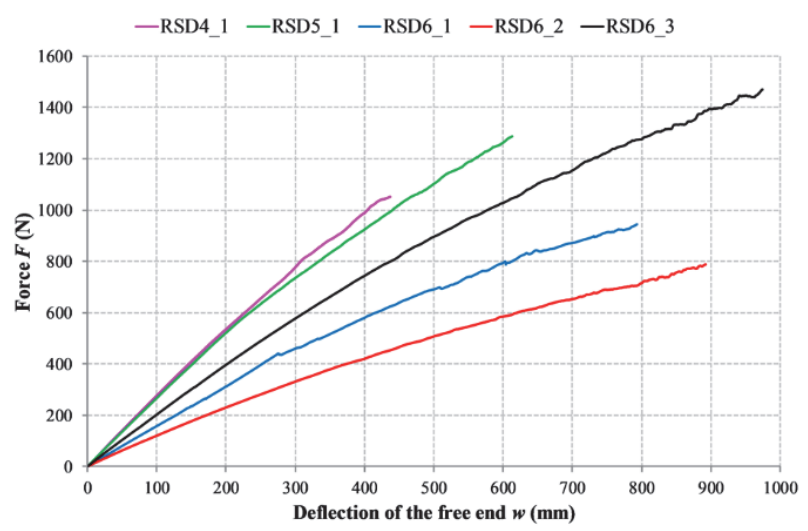

Figure 3 Force-free end deflection diagram for all GRP poles $(x=L)$

The measured values of the deflection at three measuring points related to the applied force at the free end are shown in Fig. 4 for the pole RSD6_3 as an example.

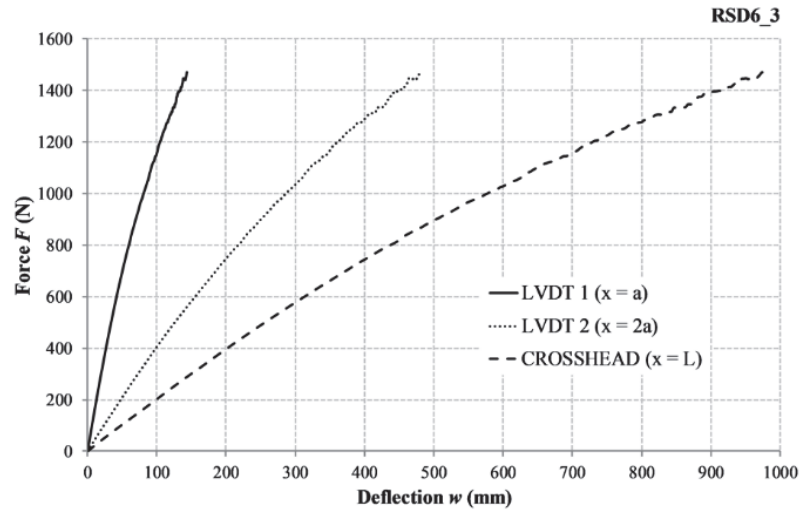

Figure 4 The force-deflection diagram at different measuring points (RSD6_3)

\subsection{Experimental Flexural Modulus Based on the Classical Beam Theory}

The displacements of orthotropic beams and composite beams with symmetrical cross sections, loaded in the symmetry plane are similar to displacements of isotropic beams [19]. For that reason, an isotropic beam of the same geometry as the composite beam with the same boundary conditions and loading as in the experimental setup in Fig. 2, was considered. Defining analytical expressions for a tapered tube with a variable wall thickness across the length represented a challenge. Based on the known dimensions of the hollow circular crosssections at the fixed and free end of the beam it was convenient to represent the variation of the outer $\left(D_{o}\right)$ and inner $\left(D_{i}\right)$ diameter across the length as linear equations.
The equations for the variation of the outer and inner diameter therefore are:

$$
\begin{aligned}
& D_{o}(x)=\frac{D_{\text {min }}-D_{\text {max }}}{L} x+D_{\text {max }} \\
& D_{i}(x)=\frac{D_{\text {min }}-D_{\text {max }}+2\left(t_{\text {min }}-t_{\text {max }}\right)}{L} x+D_{\text {max }}-2 t_{\text {min }}
\end{aligned}
$$

The equation for the moment of inertia across the length of the tube is as follows:

$I(x)=\frac{\left\{\left[D_{o}(x)\right]^{4}-\left[D_{i}(x)\right]^{4}\right\} \pi}{64}$

The outer and the inner diameter as well as the moment of inertia represent the cross-sectional properties which are used to obtain the analytical expression for the deflection line. This equation is further solved for the analytical value of the flexural modulus of elasticity at the characteristic cross-sections, i.e. the measuring points from the experimental setup. The expressions for the maximum flexural stresses and strains are also derived for these characteristic cross-sections. Finally, based on the measured values of the force and the deflection, an apparent value of the flexural modulus can be determined at characteristic cross-sections as a secant modulus. This value represents the effective flexural modulus obtained experimentally and can be further implemented in the isotropic beam solutions. The procedure of determining the flexural modulus of elasticity based on the classical beam theory is shown in Fig. 5.

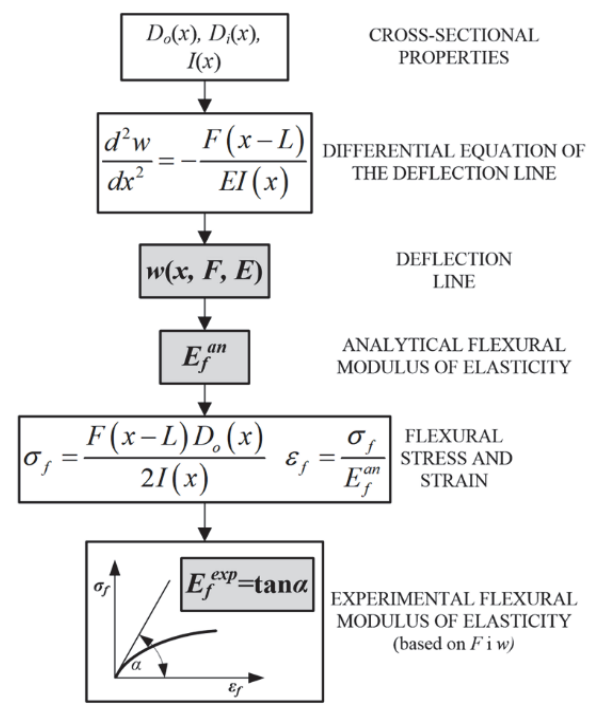

Figure 5 Determination of the flexural modulus based on the classical beam theory

\subsection{Mechanical Properties of an Orthotropic Laminate}

The GRP pole is a laminated composite, made of plies with continuous fibres embedded in a matrix by the filament winding process. A filament wound layer with the same number of plies with fibres in the $+\theta$ and $-\theta$ directions is an orthotropic material, where $\theta$ is the angle 
between the fibres and the longitudinal axis of the tube. In order to use the classical lamination theory, it is assumed that each layer is in a state of plane stress.

The building block for the mechanical analysis of a thin fibre-reinforced laminate, such as the wall of a filament wound tube, is a unidirectional (UD) ply $(\theta=0)$ under plane stress represented with four engineering constants $E_{1}, E_{2}, G_{12}$ and $v_{12}$. The reduced stiffness matrix $[Q]$ of a UD ply is obtained from the compliance matrix $[S]$ with elements in terms of the engineering constants as follows:

$$
[\boldsymbol{Q}]=[\boldsymbol{S}]^{-1}=\left[\begin{array}{ccc}
\frac{1}{E_{1}} & \frac{-v_{12}}{E_{1}} & 0 \\
\frac{-v_{12}}{E_{1}} & \frac{1}{E_{2}} & 0 \\
0 & 0 & \frac{1}{G_{12}}
\end{array}\right]^{-1}
$$

For an angle ply $(\theta \neq 0)$ the transformed reduced stiffness matrix is defined as:

$$
[\overline{\boldsymbol{Q}}]=\left[\boldsymbol{T}_{\sigma}\right]^{-1}[\boldsymbol{Q}]\left[\boldsymbol{T}_{\varepsilon}\right]
$$

using stress and strain transformation matrices with $c=$ $\cos \theta$ and $s=\sin \theta$ as:

$$
\begin{aligned}
& {\left[\boldsymbol{T}_{\sigma}\right]=\left[\begin{array}{ccc}
c^{2} & s^{2} & 2 c s \\
s^{2} & c^{2} & -2 c s \\
-c s & c s & c^{2}-s^{2}
\end{array}\right]} \\
& {\left[\boldsymbol{T}_{\varepsilon}\right]=\left[\begin{array}{ccc}
c^{2} & s^{2} & c s \\
s^{2} & c^{2} & -c s \\
-2 c s & 2 c s & c^{2}-s^{2}
\end{array}\right]}
\end{aligned}
$$

For a filament wound layer which is symmetrical and orthotropic, the stiffness matrix is calculated from the stiffness matrices of plies oriented in the $+\theta$ and $-\theta$ directions according to the following equation [19]:

$Q_{i j}^{ \pm \theta}=\frac{1}{2}\left(\bar{Q}_{i j}^{+\theta}+\bar{Q}_{i j}^{-\theta}\right), \quad i, j=1,2,6$

It is convenient to use the laminate engineering constants, i.e. effective properties which may be derived from the laminate compliance matrix. The compliance matrix of a filament wound layer $[S]^{ \pm \theta}$ is obtained by inverting the stiffness matrix $[\boldsymbol{Q}]^{ \pm \theta}$. The effective properties of a filament wound layer are further given as [25]:

$E_{x}^{ \pm \theta}=\frac{1}{S_{11}^{ \pm \theta}}$

$$
\begin{aligned}
& E_{y}^{ \pm \theta}=\frac{1}{S_{22}^{ \pm \theta}} \\
& G_{x y}^{ \pm \theta}=\frac{1}{S_{66}^{ \pm \theta}} \\
& v_{x y}^{ \pm \theta}=-\frac{S_{12}^{ \pm \theta}}{S_{11}^{ \pm \theta}}
\end{aligned}
$$

\subsection{Engineering Constants of the GRP Material}

Information on the material properties of the matrix and the fibres, layer thickness, orientation and amount of the fibres was obtained from the manufacturer. According to the manufacturer, the properties of the cured unreinforced resin are as follows: specific weight at $20^{\circ} \mathrm{C}$ $1,11 \mathrm{~g} / \mathrm{cm}^{3}$, tensile strength $50 \mathrm{MPa}$, tensile and flexural modulus $3900 \mathrm{MPa}$ and elongation at break 1,5\%. The remaining material properties of the matrix and E-glass fibres were gathered from the literature and are given in Tab. $2[19,26]$.

Table 2 Elastic constants of the matrix and the fibres

\begin{tabular}{|c|c|c|c|c|}
\hline \multicolumn{2}{|c|}{ MATRIX } & FIBRE & Min. & Max. \\
\hline$E_{m} / \mathrm{MPa}$ & 3900 & $E_{f} / \mathrm{MPa}$ & 72000 & 85000 \\
\hline$G_{m} / \mathrm{MPa}$ & 1466 & $G_{f} / \mathrm{MPa}$ & 30000 & 35417 \\
\hline$v_{m}$ & 0,33 & $v_{f}$ & 0,2 & 0,2 \\
\hline
\end{tabular}

In order to estimate the material properties of the laminate, a micromechanical approach considering fibre and matrix elastic constants was applied. The four engineering constants of a UD ply are the longitudinal and transverse Young's moduli, the shear modulus and the Poisson's ratio. They were determined according to the rule of mixtures where $V_{f}$ is the fibre volume fraction.

$$
\begin{aligned}
& E_{1}=E_{f} V_{f}+E_{m}\left(1-V_{f}\right) \\
& \frac{1}{E_{2}}=\frac{V_{f}}{E_{f}}+\frac{\left(1-V_{f}\right)}{E_{m}} \\
& \frac{1}{G_{12}}=\frac{V_{f}}{G_{f}}+\frac{\left(1-V_{f}\right)}{G_{m}} \\
& v_{12}=v_{f} V_{f}+v_{m}\left(1-V_{f}\right)
\end{aligned}
$$

\subsection{Replacement Flexural Stiffness Based on the Classical Lamination Theory}

In this study, the laminated tube consists of a layer with the layup sequence $( \pm 50)$ and a $1,2 \mathrm{~mm}$ thick finishing layer $( \pm 80)$ (Fig. 6). The midplane of a laminate is chosen as the reference plane. The wall thickness of the tube $t$ and the radius of curvature of the reference plane $R$ are varying across the pole length. 


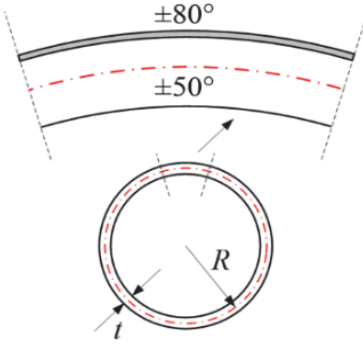

Figure 6 The cross section of the laminated tube

Thin laminates are characterized by three stiffness matrices: the extensional matrix $[\boldsymbol{A}]$, the coupling matrix $[\boldsymbol{B}]$ and the bending matrix $[\boldsymbol{D}][27]$. The expressions related to a filament wound laminate are as follows:

$A_{i j}=\sum_{k=1}^{K}\left(Q_{i j}^{ \pm \theta}\right)_{k}\left(z_{k}-z_{k-1}\right)$

$B_{i j}=\frac{1}{2} \sum_{k=1}^{K}\left(Q_{i j}^{ \pm \theta}\right)_{k}\left(z_{k}^{2}-z_{k-1}^{2}\right)$

$D_{i j}=\frac{1}{3} \sum_{k=1}^{K}\left(Q_{i j}^{ \pm \theta}\right)_{k}\left(z_{k}^{3}-z_{k-1}^{3}\right)$

For the laminated tube shown in Fig. 6, the total number of layers is $K=2$ and the distances from the reference plane to the surfaces of each layer are $z_{0}=-t / 2$, $z_{1}=-t / 2+1,2$ and $z_{2}=t / 2$.

For symmetrical laminates, the coupling matrix $[\boldsymbol{B}]=$ 0 and the compliance matrices of a laminate $[a]$ and $[d]$ are obtained by directly inverting the matrices $[\boldsymbol{A}]$ and $[\boldsymbol{D}]$, respectively. For unsymmetrical laminates, the compliance matrices $[\alpha],[\beta]$ and $[\delta]$ are obtained from the stiffness matrices as follows:

$\left[\begin{array}{cc}\boldsymbol{\alpha} & \boldsymbol{\beta} \\ \boldsymbol{\beta}^{T} & \boldsymbol{\delta}\end{array}\right]=\left[\begin{array}{ll}\boldsymbol{A} & \boldsymbol{B} \\ \boldsymbol{B} & \boldsymbol{D}\end{array}\right]^{-1}$

The elements of the compliance matrices can further be used to calculate the replacement stiffnesses [19]. For a doubly symmetrical hollow circular cross section with an orthotropic and unsymmetrical layup of the wall segment, the replacement flexural stiffness is defined as:

$\hat{E} I_{y}=\hat{E} I_{z}=\pi\left(\frac{R^{3}}{\alpha_{11}}+\frac{R}{\delta_{11}}\right)$

The properties $\alpha_{11}$ and $\delta_{11}$ are evaluated at each wall segment's "neutral" plane using the properties related to the midplane (MP) as follows [19]:

$\alpha_{11}=\alpha_{11}^{\mathrm{MP}}+2 \tilde{\rho} \beta_{11}^{\mathrm{MP}}+\tilde{\rho}^{2} \delta_{11}^{\mathrm{MP}}$

$\delta_{11}=\delta_{11}^{\mathrm{MP}}$
The "neutral" plane is located at a distance $\tilde{\rho}$ from the reference plane [19].

$\tilde{\rho}=-\frac{\beta_{11}^{\mathrm{MP}}}{\delta_{11}^{\mathrm{MP}}}$

\subsubsection{An Overview of the Method}

The method of determining the replacement flexural stiffness of a laminated tube based on the lamination theory is shown graphically in Fig. 7. The stiffness and compliance matrices of a filament wound laminate were calculated in SageMath according to the equations in Section 2.5. It is necessary to emphasize that the replacement flexural stiffness was determined at the characteristic cross-sections, i.e. the measuring points from the experimental setup.

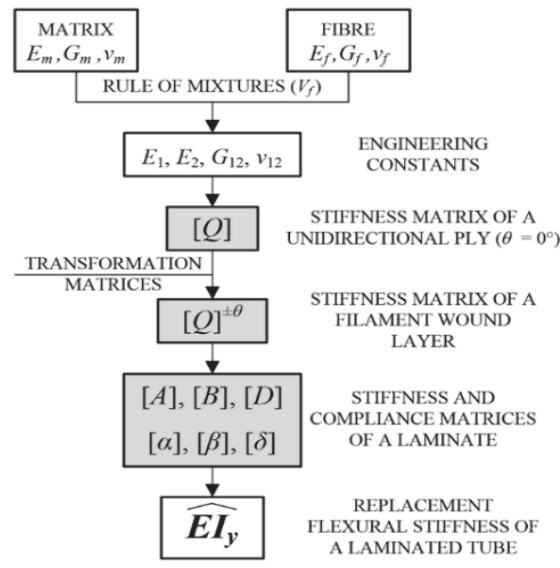

Figure 7 Determination of the replacement flexural stiffness based on the lamination theory

\section{RESULTS}

\subsection{Determination of the Experimental Flexural Modulus}

The variation of the moment of inertia across the length for all tested poles according to the Eq. (3) is shown in Fig. 8.

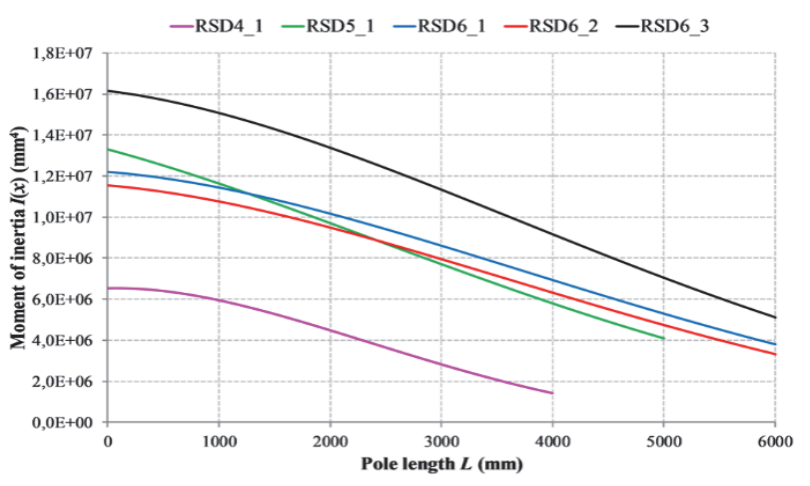

Figure 8 Moment of inertia across the pole length

The cross-sectional properties and the boundary conditions of a cantilever beam are used to obtain the analytical expression for the deflection line according to the classical beam theory. Based on the slope of the loaddeflection curve, the analytical flexural modulus of elasticity $E_{f}^{a n}$ is derived at the characteristic cross-sections 
(Tab. 3). The characteristic cross-sections of each pole are the measuring points from the experimental setup.

Table 3 The analytical flexural modulus at the characteristic cross-sections

\begin{tabular}{|c|c|c|}
\hline \multirow{2}{*}{ Pole designation } & \multicolumn{2}{|c|}{$E_{f}^{a n} / \mathrm{MPa}$} \\
\cline { 2 - 3 } & $x=a$ & $x=2 a$ \\
\hline RSD4_1 & $621,741 \cdot F / w$ & $2314,06 \cdot F / w$ \\
\hline RSD5_1 & $708,03 \cdot F / w$ & $2631,39 \cdot F / w$ \\
\hline RSD6_1 & $911,166 \cdot F / w$ & $3396,58 \cdot F / w$ \\
\hline RSD6_2 & $964,592 \cdot F / w$ & $3606,87 \cdot F / w$ \\
\hline RSD6_3 & $689,708 \cdot F / w$ & $2573,71 \cdot F / w$ \\
\hline
\end{tabular}

Using analytical expressions of the flexural modulus $E_{f}^{a n}$ as well as the measured values of the force and the deflection, the calculation of the maximum flexural stresses and strains was performed according to Fig. 5. The results are similar for all poles and are shown in Fig. 9 for the pole RSD6_3.

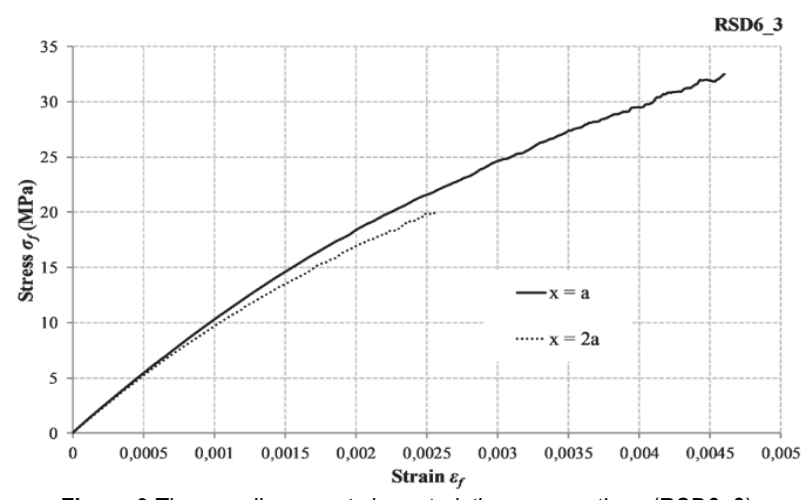

Figure 9 The $\sigma_{f-\varepsilon_{f}}$ diagram at characteristic cross sections (RSD6_3)

Apparent values of the flexural modulus $E_{f}^{\exp }$ were determined for the characteristic cross-sections as a secant modulus at the strain value of $\varepsilon_{f}=0,0005$ (Tab. 4). This strain range corresponds to the linear stress/strain behaviour (Fig. 9). The same range is relevant for the determination of flexural properties of FRP composites under three-point and four-point bending test with smaller specimens [7].

Table 4 The experimental flexural modulus at the characteristic cross-sections

\begin{tabular}{|c|c|c|c|}
\hline \multirow{2}{*}{ Pole designation } & \multicolumn{3}{|c|}{$E_{f}^{\exp } / \mathrm{MPa}$} \\
\cline { 2 - 4 } & $x=a$ & $x=2 a$ & Average \\
\hline RSD4_1 & 10447 & 10452 & 10449 \\
\hline RSD5_1 & 10137 & 9966 & 10051 \\
\hline RSD6_1 & 11119 & 10513 & 10816 \\
\hline RSD6_2 & 8877 & 8310 & 8594 \\
\hline RSD6_3 & 10871 & 10473 & 10672 \\
\hline
\end{tabular}

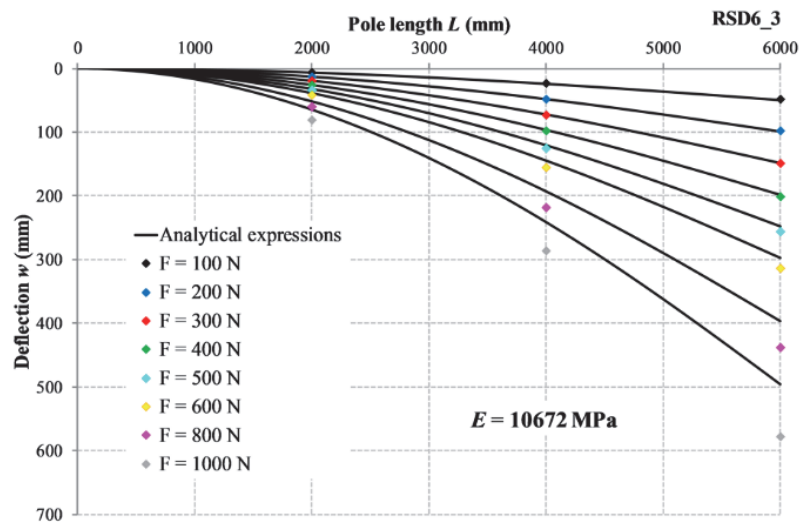

Figure 10 The deflection lines for different loading phases (RSD6_3)
The average values of the experimental flexural modulus $E_{f}^{\exp }$ are further used to finalize the analytical expressions for the deflection lines. Analytical expressions are compared to measured values at three measuring points for different loading phases in Fig. 10 (pole RSD6_3). The results are similar for all other poles.

\subsection{Sensitivity Analysis of the Laminate Material Properties}

For polymeric composites, the fibre-to-matrix moduli ratio is very high [28]. For high values of the $E_{f} / E_{m}$ ratio, the contribution of the fibre modulus $E_{f}$ to composite moduli $E_{2}$ and $G_{12}$ only increases substantially for a fibre volume fraction greater than $80 \%$ which is seldom the case $[28,29]$. The Poisson's ratios of the fibre and the matrix have little effect on the composite moduli for practical values of the Poisson's ratios [30].

The material properties of the matrix and the fibres from Tab. 2 were used to calculate the engineering constants of a UD ply using the rule of mixtures Eq. (13) to Eq. (16). The values were further normalized to the known matrix properties and plotted as a function of the fibre volume fraction in Fig. 11. For the fibre volume fraction $V_{f}=60 \%$ and the maximum value of $E_{f}$, the values of the transverse modulus $E_{2}$, as well as the shear modulus $G_{12}$ increase by $1 \%$. The contribution of the fibre modulus $E_{f}$ to the longitudinal modulus of the composite $E_{1}$ is substantial even for lower values of $V_{f}$. For the fibre volume fraction $V_{f}=60 \%$ and the maximum value of $E_{f}$, the value of $E_{1}$ increases by $17 \%$. On the other hand, a difference in the fibre volume fraction of $\pm 5 \%$ results in a difference of $+13 \%$ and $-10 \%$ for $E_{2}\left(G_{12}\right)$ and $\pm 8 \%$ for $E_{1}$.

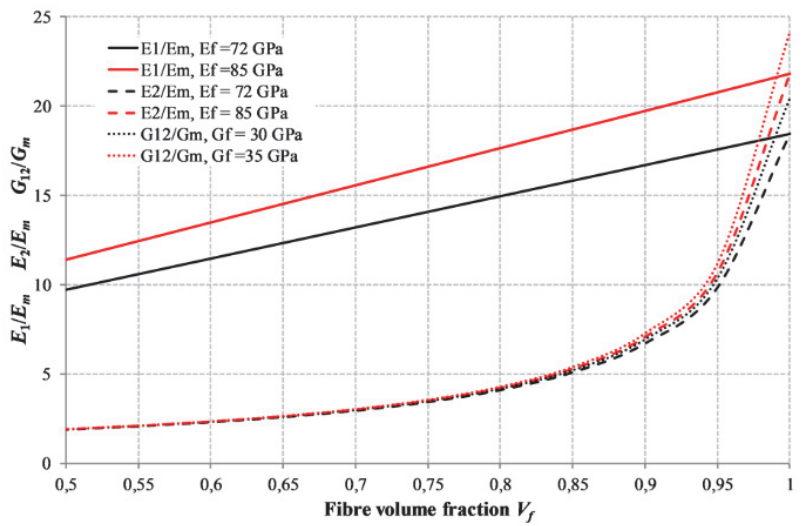

Figure 11 Normalized engineering constants of a UD ply plotted as a function of

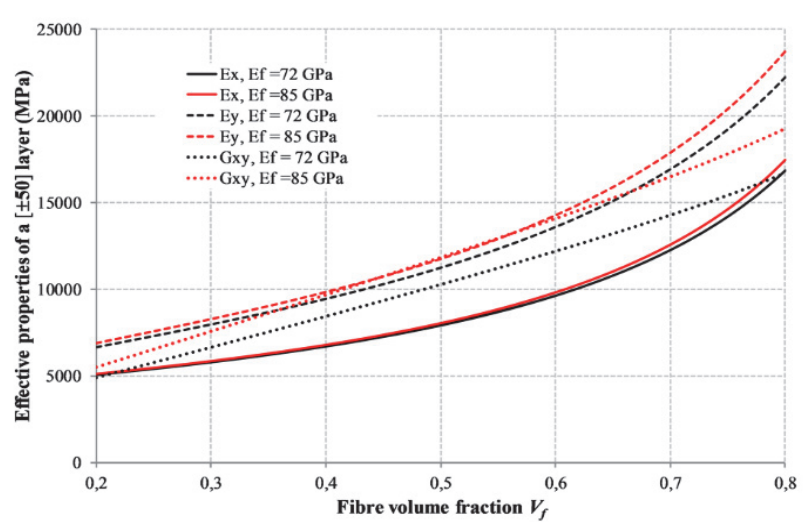

Figure 12 Effective properties of a filament wound layer $( \pm 50)$ 
The effective properties of a filament wound layer can be obtained from the elements of the compliance matrix as was shown with the Eq. (9) to Eq. (12). The calculated values for the $( \pm 50)$ layer are plotted as a function of the fibre volume fraction in Fig. 12.

With the maximum value of $E_{f}$, the values of the longitudinal modulus $E_{x}$, the transverse modulus $E_{y}$ and the shear modulus $G_{x y}$ of the filament wound layer increase by $2 \%, 5 \%$ and $16 \%$, respectively. Thus, the longitudinal modulus is a matrix dominated property and the shear modulus is a fibre dominated property [31]. On the other hand, a difference in the fibre volume fraction of $\pm 5 \%$ results in a difference of up to $+12 \%$ and $-10 \%$ for all moduli.

Since the exact values of the fibre volume fraction for different tubes were not determined experimentally, it was necessary to consider several combinations of the engineering constants with a difference in the fibre volume fraction of $\pm 5 \%$ (Tab. 5). Even though the minimum value of the fibre modulus $E_{f}=72 \mathrm{GPa}$ is more accurate for Eglass fibres, the maximum value was also used in further analysis.

Table 5 Engineering constants of a UD ply

\begin{tabular}{|c|c|c|c|c|c|c|}
\hline Laminate & $0.55 \quad 72$ & 0.5585 & $0.60 \quad 72$ & 0.6085 & $0.65 \quad 72$ & $0.65 \quad 85$ \\
\hline$E_{f} / E_{m}$ & 18,5 & 21,8 & 18,5 & 21,8 & 18,5 & 21,8 \\
\hline$G_{f} / G_{m}$ & 20 & 24 & 20 & 24 & 20 & 24 \\
\hline$E_{1} / \mathrm{MPa}$ & 41355 & 48505 & 44760 & 52560 & 48165 & 56615 \\
\hline$E_{2} / \mathrm{MPa}$ & 8129 & 8206 & 9017 & 9122 & 10124 & 10268 \\
\hline$G_{12} / \mathrm{MPa}$ & 3074 & 3101 & 3415 & 3451 & 3840 & 3890 \\
\hline$v_{12}$ & 0,26 & 0,26 & 0,25 & 0,25 & 0,25 & 0,25 \\
\hline$V_{f}$ & \multicolumn{2}{|c|}{0,55} & \multicolumn{2}{|c|}{0,60} & \multicolumn{2}{|c|}{0,65} \\
\hline
\end{tabular}

\subsection{Determination of the Replacement Flexural Stiffness of the Laminated Tube}

The procedure of determining the replacement flexural stiffness was further carried out according to Fig. 7 for six combinations of engineering constants of a UD ply from Tab. 5. For example, the stiffness matrix of a UD ply $(\theta=$ 0 ) for the laminate $0.6 \_72$ is:

$$
[Q]=\left[\begin{array}{ccc}
45331 & 2283 & 0 \\
2283 & 9132 & 0 \\
0 & 0 & 3415
\end{array}\right] \mathrm{MPa}
$$

and the stiffness matrices for both filament wound layers are:

$$
\begin{aligned}
& {\left[Q^{ \pm 50}\right]=\left[\begin{array}{ccc}
15302 & 11069 & 0 \\
11069 & 21588 & 0 \\
0 & 0 & 12201
\end{array}\right] \mathrm{MPa}} \\
& {\left[Q^{ \pm 80}\right]=\left[\begin{array}{ccc}
9164 & 3343 & 0 \\
3343 & 43180 & 0 \\
0 & 0 & 4475
\end{array}\right] \mathrm{MPa}}
\end{aligned}
$$

The stiffness and compliance matrices of the laminate $[ \pm 50 / \pm 80]$ were further calculated considering the geometry of each pole at characteristic cross-sections $x=a$ and $x=2 a$ according to Eq. (17) to Eq. (20). The elements of the compliance matrices $\alpha_{11}$ and $\delta_{11}$ considering the orthotropic and unsymmetrical layup of the wall segment were used to calculate the replacement flexural modulus according to Eq. (21). The results of the analysis for all

\begin{tabular}{|c|c|c|c|c|}
\hline $\begin{array}{c}\text { Pole } \\
\text { designation }\end{array}$ & $\begin{array}{c}\text { Cross } \\
\text { section }\end{array}$ & $\alpha_{11} / \mathrm{mm} / \mathrm{N}$ & $\delta_{11} / 1 / \mathrm{Nmm}$ & $\hat{E} / \mathrm{MPa}$ \\
\hline \multirow{2}{*}{ RSD4_1 } & $x=a$ & $10,851 \times 10^{-6}$ & $14,626 \times 10^{-7}$ & 9920 \\
\hline & $x=2 a$ & $7,7316 \times 10^{-6}$ & $5,2524 \times 10^{-7}$ & 9788 \\
\hline \multirow{2}{*}{ RSD5_1 } & $x=a$ & $7,6155 \times 10^{-6}$ & $5,0176 \times 10^{-7}$ & 9874 \\
\hline & $x=2 a$ & $6,1439 \times 10^{-6}$ & $2,6230 \times 10^{-7}$ & 9740 \\
\hline \multirow{2}{*}{ RSD6_1 } & $x=a$ & $8,8627 \times 10^{-6}$ & $7,9350 \times 10^{-7}$ & 9911 \\
\hline & $x=2 a$ & $6,8642 \times 10^{-6}$ & $3,6661 \times 10^{-7}$ & 9815 \\
\hline \multirow{2}{*}{ RSD6_2 } & $x=a$ & $9,3822 \times 10^{-6}$ & $9,4254 \times 10^{-7}$ & 9918 \\
\hline & $x=2 a$ & $7,2355 \times 10^{-6}$ & $4,2986 \times 10^{-7}$ & 9831 \\
\hline \multirow{2}{*}{ RSD6_3 } & $x=a$ & $7,1843 \times 10^{-6}$ & $4,2074 \times 10^{-7}$ & 9872 \\
\hline & $x=2 a$ & $5,6045 \times 10^{-6}$ & $1,9875 \times 10^{-7}$ & 9738 \\
\hline
\end{tabular}
poles considering the laminate $0.6 \_72$ are shown as an example in Tab. 6.

The wall thickness of the tapered laminated tube $t$ and the radius of curvature of the reference plane $R$ are linearly varying across the pole length which is shown for the pole RSD6 3 in Fig. 13. In order to investigate the influence of the variable wall thickness, the replacement flexural modulus was calculated for different cross-sections across the pole length considering the laminate 0.6_72 (Fig. 13). Since the layer thickness was not determined experimentally, the procedure was also repeated without the $( \pm 80)$ finishing layer as well as with a single and a double finishing layer throughout the wall thickness. The values have a slightly decreasing tendency towards the free end of the pole which is in accordance with the experimental results considering the two characteristic cross-sections. Adding a second finishing layer causes a minor effect on the values of the replacement flexural modulus in comparison with the wall thickness entirely consisted of a $( \pm 50)$ laminate (no finishing layer).

The results for all poles considering six material combinations at the cross-section $x=a$ are shown in Fig. 14 and at the cross-section $x=2 a$ in Fig. 15. With the maximum value of $E_{f}$, the values of the replacement flexural modulus increase by up to $3 \%$. A difference in the fibre volume fraction of $\pm 5 \%$ results in a difference of $+12 \%$ and $-10 \%$. The results are in accordance with the effective properties of a filament wound layer $( \pm 50)$ described in Section 3.2.

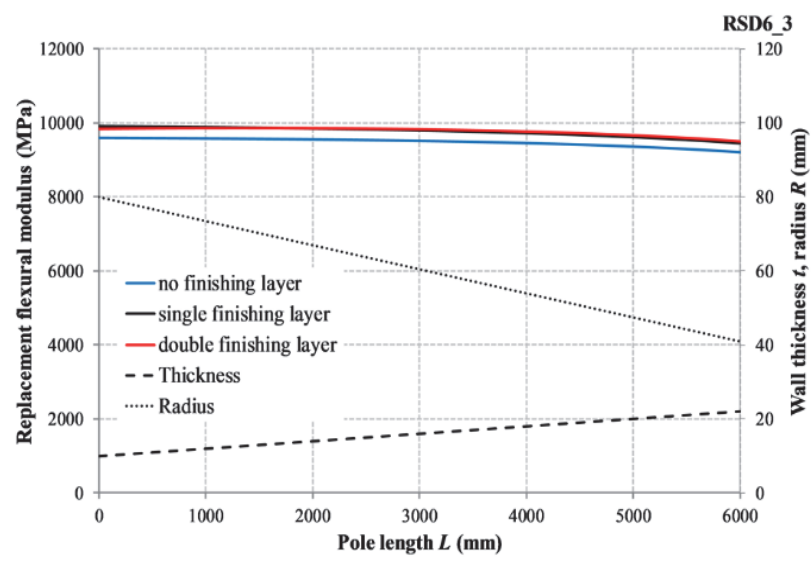

Figure 13 Replacement flexural modulus across the pole length 


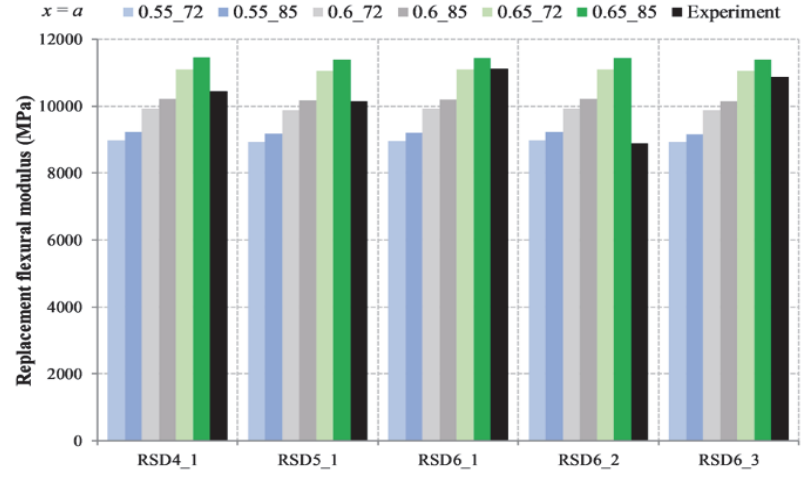

Figure 14 Replacement flexural modulus at the $x=a$ cross-section

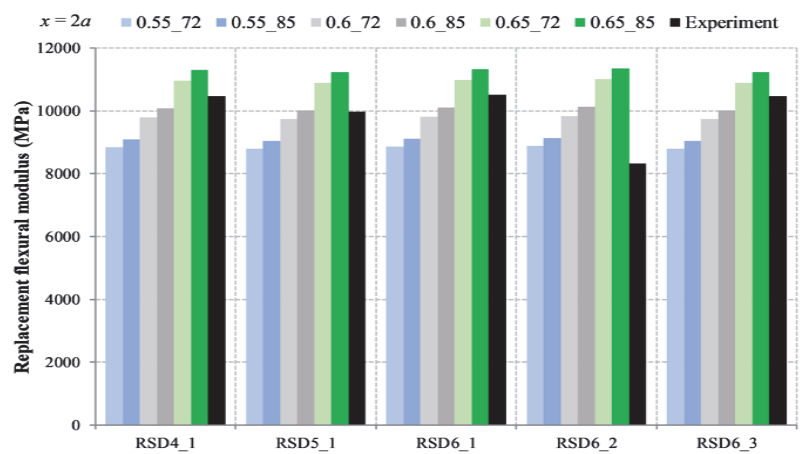

Figure 15 Replacement flexural modulus at the $x=2 a$ cross-section

Finally, the differences between the theoretical values obtained according to the classical lamination theory and the experimental values obtained based on the classical beam theory are summarized in Tab. 7 for the $x=a$ crosssection. Since the flexural modulus is a matrix dominated property, the analysis performed with the relevant minimum value of the fibre modulus $E_{f}=72 \mathrm{GPa}$ is presented, which is more accurate for E-glass fibres. The average differences between the experimental and the theoretical values considering the $0.55 \_72,0.6 \_72$ and 0.65 72 laminate are $-12,5 \%,-3,2 \%$ and $8,3 \%$, respectively. The results show that it is necessary to determine the accurate value of the fibre volume fraction since even minor variations $( \pm 5 \%)$ from the average value of the volume fraction $(0,6)$ cause considerable changes in the values of the replacement flexural modulus.

Table 7 Differences between theoretical and experimental values

\begin{tabular}{|c|c|c|c|}
\hline$x=a$ & \multicolumn{3}{|c|}{$\hat{E} / \mathrm{MPa}$} \\
\hline Pole designation & $0.55 \_72$ & $0.6 \_72$ & 0.65772 \\
\hline RSD4_1 & 8968 & 9920 & 11092 \\
\hline$E_{f}^{\text {exp }}=10447 \mathrm{MPa}$ & $-14 \%$ & $-5 \%$ & $6 \%$ \\
\hline RSD5_1 & 8925 & 9874 & 11043 \\
\hline$E_{f}^{\text {exp }}=10137 \mathrm{MPa}$ & $-12 \%$ & $-3 \%$ & $9 \%$ \\
\hline RSD6_1 & 8959 & 9911 & 11083 \\
\hline$E_{f}^{\text {exp }}=11119 \mathrm{MPa}$ & $-19 \%$ & $-11 \%$ & $0 \%$ \\
\hline RSD6_2 & 8966 & 9918 & 11091 \\
\hline$E_{f}^{\text {exp }}=8877 \mathrm{MPa}$ & $1 \%$ & $12 \%$ & $25 \%$ \\
\hline RSD6_3 & 8924 & 9872 & 11042 \\
\hline$E_{f}^{\text {exp }}=10871 \mathrm{MPa}$ & $-18 \%$ & $-9 \%$ & $2 \%$ \\
\hline Average difference & $-12,5$ & $-3,2$ & 8,3 \\
\hline Standard deviation & 9,4 & 8,3 & 9,3 \\
\hline
\end{tabular}

\section{DISCUSSION}

In the focus of this research are the flexural properties of composite lighting poles which are GRP tapered tubes. The poles are manufactured by the filament winding process. In this study, a cantilever flexural test was carried out on full-scale GRP tubes in order to determine the effective flexural modulus. During lateral loading at the free end of the pole, the applied force was measured as well as the deflection at three different measuring points. By analogy with the three-point and four-point flexural tests of FRP composites, flexural properties were determined based on the classical beam theory. Considering the cantilever beam, the $\sigma_{f}-\varepsilon_{f}$ curve was plotted for the two cross-sections within the span of each pole. The results show that the strain range corresponding to the linear $\sigma_{f}$ $\varepsilon_{f}$ behaviour is the same as under three-point and four-point loading with smaller specimens. An average value of the flexural modulus from the two cross-sections was implemented in the derived analytical expression for the deflection line. A very good agreement was achieved between measured values and analytical curves under lower levels of loading. Unlike other methods, the proposed method of determining the effective flexural modulus based on experimental results considers the variation of the cross-sectional properties across the pole length. Without dividing the length of the pole into zones with average properties, a single analytical expression for the deflection line was developed.

The validated values of the flexural modulus based on the classical beam theory and experimental results were further compared to the predicted values of the effective flexural properties based on the CLT. The analysis was performed for the two characteristic cross-sections within the span of each pole. The wall of the GRP pole is made of orthotropic filament wound layers and the effective properties are highly influenced by the material properties of the matrix and the fibres, the amount and orientation of the fibres as well as the layer thickness. Information on the material properties of the matrix and the fibres, layer thickness, orientation and amount of the fibres was obtained from the manufacturer and the literature. The effective properties were determined at the lamina, laminate and structure level.

First, a UD ply was considered, and the engineering constants were determined using the rule of mixtures by assuming a minimum and a maximum value of the fibre modulus. Even though the minimum value of the fibre modulus is more accurate for E-glass fibres, the maximum value was also used in the analysis. The values were further normalized to the known matrix properties and plotted as a function of the fibre volume fraction. It is confirmed that the transverse modulus $E_{2}$, as well as the shear modulus $G_{12}$ are matrix dominated and that the contribution of the fibre modulus $E_{f}$ to the longitudinal modulus of the composite $E_{1}$ is substantial even for lower values of the volume fraction $V_{f}$. A difference in the fibre volume fraction of $\pm 5 \%$ results in a difference of $+13 \%$ and $-10 \%$ for $E_{2}\left(G_{12}\right)$ and $\pm 8 \%$ for $E_{1}$.

A further analysis was performed for the $( \pm 50)$ filament wound layer. It is confirmed that the longitudinal modulus $E_{x}$ of a filament wound layer is a matrix dominated property and the shear modulus $G_{x y}$ is a fibre dominated property. A difference in the fibre volume fraction of $\pm 5 \%$ results in a difference of up to $+12 \%$ and $-10 \%$ for all moduli which is similar as for the UD ply.

Next, the effective (replacement) flexural stiffness of a filament wound tube with an orthotropic and 
unsymmetrical layup of the wall segment was determined based on the CLT. The stiffness and compliance matrices of the laminate $( \pm 50 / \pm 80)$ were calculated, considering the geometry of each pole at the characteristic cross-sections. Since the $( \pm 80)$ is only a finishing layer and the thickness of each layer was not determined experimentally, the influence of the variable wall thickness and the thickness of the finishing layer was investigated. The values of the replacement flexural modulus have a slightly decreasing tendency towards the free end of the pole which is in accordance with the experimental results considering the two characteristic cross-sections. By adding a second finishing layer under constant wall thickness, a minor effect on the values of the replacement flexural modulus is noticed.

The values of the replacement flexural modulus for all poles considering six material combinations increase by up to $3 \%$ with the maximum value of $E_{f}$. A difference in the fibre volume fraction of $\pm 5 \%$ results in a difference of $+12 \%$ and $-10 \%$. The results correspond to the effective properties of a filament wound layer $( \pm 50)$ and it can be concluded that the replacement flexural modulus is a matrix dominated property.

Finally, the differences between the theoretical values obtained according to the CLT and the experimental values obtained based on the classical beam theory show that it is necessary to determine the accurate value of the fibre volume fraction. Even minor variations $( \pm 5 \%)$ from the average value of the fibre volume fraction cause considerable changes in the values of the replacement flexural modulus.

\section{CONCLUSIONS}

The effective properties of FRP materials can be used to develop material models in the numerical analysis or to simplify the complex mechanical behaviour of laminated composites. The latter is achieved by replacing the isotropic stiffnesses with the effective (replacement) stiffnesses in the corresponding isotropic beam solutions. In this paper, the effective flexural properties of a filament wound tapered pole with a variable wall thickness were determined, experimentally and theoretically. The first method is based on the analogy with the widely accepted three-point and four-point flexural tests with small test specimens. The flexural modulus is calculated from the initial slope of the $\sigma_{f}-\varepsilon_{f}$ curve based on the classical beam theory. The replacement flexural stiffness was also derived based on the classical lamination theory incorporating material properties of the matrix and the fibres, layer thickness as well as the amount and orientation of the fibres. Information on these parameters is often insufficient and experimental testing is needed in order to accurately determine the effective properties. The results from this study indicate possible strengths and weaknesses of predicting the effective properties. Since the effective flexural modulus is a matrix dominated property it is necessary to obtain the accurate material properties of the matrix, experimentally or from the manufacturer. Also, an accurate value of the fibre volume fraction is of great significance since even minor variations from the average value cause considerable changes in the values of the replacement flexural modulus. Further research should include an experimental investigation of the fibre volume fraction considering poles with different thicknesses and lengths. In this way, the methodology can be further checked and upgraded.

\section{REFERENCES}

[1] Tipton, B. (2008). Prevention of Environmentally Induced Degradation in Carbon/epoxy Composite Material via Implementation of a Polymer Based Coating System. Thesis. University of Central Florida.

[2] HRN EN 40-7:2008. Lighting columns-Part 7: Requirements for fibre reinforced polymer composite lighting columns.

[3] ANSI C136.20-2012. American National Standard For Roadway And Area Lighting Equipment-Fiber-Reinforced Composite (FRC) Lighting Poles.

[4] Polyzois, D, Raftoyiannisz, I. G., \& Philopulos, D. (2007). An Experimental Survey of the Static and Dynamic Behavior of Jointed Composite GFRP Tapered Poles. Mech. Adv. Mater. Struct., 14, 203-212. https://doi.org/10.1080/15376490600734849

[5] Sideridis, E. \& Papadopoulos, G. A. (2004). Short-Beam and Three-Point-Bending Tests for the Study of Shear and Flexural Properties in Unidirectional-Fiber-Reinforced Epoxy Composites. J. Appl. Polym. Sci., 93, 63-74. https://doi.org/10.1002/app.20382

[6] Bondyra, A., Klasztorny, M., Szurgott, P., et al. (2012). Numerical Modelling and Experimental Verification of Glass-Polyester Mixed Laminate Beam Bending Test. Acta Mech. Autom., 6.

[7] HRN EN ISO 14125:2005. Fibre-reinforced plastic composites-Determination of flexural properties.

[8] Berube, K. A., Lopez-Anido, R. A., \& Goupee, A. J. (2016). Determining the Flexural and Shear Moduli of FiberReinforced Polymer Composites Using Three-Dimensional Digital Image Correlation. Exp. Tech., 40, 1263-1273. https://doi.org/10.1007/s40799-016-0127-4

[9] Tavakoldavani, K., Leboulluec, P., \& Chan, W. (2015). Equivalent Mechanical and Thermal Properties of Composite Materials in Lamina, Laminate and Structure Levels. In: Xiao X, Loos AC, Liu D, editors. Proc. Am. Soc. Compos. THIRTIETH Tech. Conf. East Lansing, MI, 28-30 September 2015, 187-202.

[10] Polyzois, D., Raftoyiannis, I. G., \& Ibrahim, S. (1998). Finite elements method for the dynamic analysis of tapered composite poles. Compos. Struct., 43, 25-34. https://doi.org/10.1016/S0263-8223(98)00088-9

[11] Chan, W. S. \& Demirhan, K. C. (2000). A Simple ClosedForm Solution of Bending Stiffness for Laminated Composite Tubes. J. Reinf. Plast. Compos., 19, 278-291. https://doi.org/10.1106/Y1H1-25TK-1M0J-WJ2X

[12] Kollar, L. P. \& Pluzsik, A. (2002). Analysis of Thin-Walled Composite Beams with Arbitrary Layup. J. Reinf. Plast. Compos., 21, 1423-1465. https://doi.org/10.1177/0731684402021016928

[13] Ibrahim, S. \& Polyzois, D. (1999). Ovalization analysis of fiber-reinforced plastic poles. Compos. Struct., 45, 7-12. https://doi.org/10.1016/S0263-8223(98)00137-8

[14] Shadmehri, F., Derisi, B., \& Hoa, S. V. (2011). On bending stiffness of composite tubes. Compos. Struct., 93, 21732179. https://doi.org/10.1016/j.compstruct.2011.03.002

[15] Metiche, S. \& Masmoudi, R. (2013). Analysis and design procedures for the flexural behavior of glass fiber-reinforced polymer composite poles. J. Compos. Mater., 47, 207-229. https://doi.org/10.1177/0021998312438721

[16] Masmoudi, R., Mohamed, H., \& Metiche, S. (2008). Finite Element Modeling for Deflection and Bending Responses of GFRP Poles. J. Reinf. Plast. Compos., 27, 639-658. https://doi.org/10.1177/0731684407083009 
[17] Saboori, B. \& Khalili, S. M. R. (2011). Static analysis of tapered FRP transmission poles using finite element method. Finite Elem. Anal. Des., 47, 247-255. https://doi.org/10.1016/j.finel.2010.10.002

[18] Urgessa, G. \& Mohamadi, S. (2016). Structural Assessment of Fiber-reinforced Polymer Composite Electric Poles. Procedia Eng., 707-714. https://doi.org/10.1016/.jproeng.2016.04.085

[19] Kollar, L. P. (2003). Mechanics of composite structures. Cambridge University Press. https://doi.org/10.1017/CBO9780511547140

[20] Raftoyiannis, I. G. \& Polyzois, D. J. (2007). The effect of semi-rigid connections on the dynamic behavior of tapered composite GFRP poles. Compos. Struct., 81, 70-79. https://doi.org/10.1016/j.compstruct.2006.07.015

[21] Khalili, S. M. R. \& Saboori, B. (2010). Transient dynamic analysis of tapered FRP composite transmission poles using finite element method. Compos. Struct., 92, 275-283. https://doi.org/10.1016/j.compstruct.2009.07.026

[22] Fam, A., Kim, Y. J., \& Son, J. K. (2010). A numerical investigation into the response of free end tubular composite poles subjected to axial and lateral loads. Thin-Walled Struct., 48, 650-659. https://doi.org/10.1016/j.tws.2010.04.002

[23] Henry, T. C., Bakis, C. E., \& Smith, E. C. (2015), Determination of Effective Ply-level Properties of Filament Wound Composite Tubes Loaded in Compression. J. Test. Eval., 43, 20130159. https://doi.org/10.1520/JTE20130159

[24] Masmoudi, R. \& Metiche, S. (2007). Full-Scale Flexural Testing on Fiber-Reinforced Polymer (FRP) Poles. Open Civ. Eng. J., 1, 37-50. https://doi.org/10.2174/1874149500701010037

[25] Czél, G. \& Czigány, T. (2014). Finite element method assisted stiffness design procedure for non-circular profile composite wastewater pipe linings. Compos. Struct., 112, 78-84. https://doi.org/10.1016/j.compstruct.2014.02.003

[26] Chawla, K. K. (2012). Composite materials: science and engineering. 3rd ed. Springer.

[27] Hyer, M. W. \& White, S. R. (2009). Stress analysis of fiberreinforced composite materials. DEStech Publications, Inc.

[28] Kaw, A. K. (2006). Mechanics of composite materials. 2nd ed. Taylor \& Francis. https://doi.org/10.1201/9781420058291

[29] Gibson, R. F. (2016). Principles of composite material mechanics. 4th ed. CRC Press. https://doi.org/10.1201/b19626

[30] Jones, R. M. (1999). Mechanics of composite materials. 2nd ed. Taylor \& Francis.

[31] Daniel, I. M. \& Ishai, O. (2006). Engineering mechanics of composite materials. Oxford University Press.

\section{Contact information:}

Ana SKENDER, PhD, Assistant Professor

(Corresponding author)

University of Zagreb,

Faculty of Civil Engineering,

Fra Andrije Kačića-Miošića 26, 10000 Zagreb, Croatia

E-mail: ana.skender@grad.unizg.hr

Zoran DOMITRAN, PhD, Assistant Professor

University of Zagreb,

Faculty of Mechanical Engineering and Naval Architecture,

Ivana Lučića 5, 10000 Zagreb, Croatia

E-mail: zoran.domitran@fsb.hr

\section{Jakov KROKAR, mag. ing. aedif.}

University of Zagreb,

Faculty of Civil Engineering

Fra Andrije Kačića-Miošića 26, 10000 Zagreb, Croatia

E-mail: jakov.krokar@gmail.com 